\title{
La acción tutorial en una carrera de la universidad tecnológica
}

\author{
María del Rocío Flores Licón \\ Adolfo Valles Chávez \\ Martha Lina Castillo Pérez \\ Universidad Tecnológica de Chihuahua
}

\section{Resumen}

1 1 objetivo de la presente investigación es demostrar la importancia de la acción tutorial dentro del proceso de estadía en la Carrera de Mantenimiento Área Industrial de la Universidad Tecnológica de Chihuahua, con el fin de lograr el índice de titulación que marca la Coordinación General de Universidades Tecnológicas y Politécnicas, dentro del Modelo de Evaluación de la Calidad del Subsistema de UT. Por resultados obtenidos en investigación anterior, se decide estudiar a 139 alumnos en período de estadía en la generación 2011-2013 para comparar dichos resultados y dar continuidad a una de las líneas de investigación del cuerpo académico "Análisis Estadístico de Índices Educativos". La pregunta de investigación es: ¿Influye en el índice de titulación la acción tutorial durante el período de estadía? El diseño metodológico utilizado en este estudio es descriptivo, cuantitativo no experimental.

Palabras clave: acción tutorial, estadía, índice de titulación.

\section{Introducción}

El desempeño de las Universidades Tecnológicas se mide por el Modelo de Evaluación de la Calidad del Subsistema de Universidades Tecnológicas (MECAsut) en el cual existen varios parámetros; uno de ellos es el índice de titulación, es importante alcanzar dicho indicador porque las Universidades Tecnológicas (UT), fueron diseñadas con la finalidad de titular al 100\% de los egresados (MECASUT, 2003). La presente investigación se lleva a cabo con el objetivo 
de demostrar la importancia de la tutoría dentro del proceso de la estadía con el fin de lograr el indicador de titulación que marca la Coordinación General de Universidades Tecnológicas y Politécnicas (CGUTYP), para lo cual se pretende monitorear durante el proceso de estadía a 139 alumnos de la generación 2011-2013 de la carrera de Mantenimiento Área Industrial de la Universidad Tecnológica de Chihuahua.

Para titularse como tsu los alumnos de la UTCH, necesitan cursar el sexto cuatrimestre dentro de una empresa, este período se denomina estadía, en el cual se desarrolla un proyecto de mejora adecuado a su carrera. Para realizar este proyecto, el alumno cuenta con el apoyo de dos tutores, uno por parte de la universidad y otro por la empresa.

El cuerpo académico "Análisis Estadísticos de Índices Educativos" en el año 2010 estudió el índice de titulación de la generación 2007-2009 de Tsu, el resultado fue que el acompañamiento por parte del tutor en el proceso de estadía es de vital importancia, ya que el $92.3 \%$ de los egresados contestó que el tutor sugiere, orienta, motiva y ayuda durante la estadía a los alumnos y sólo el 7.7\% indicó que no recibió sugerencias, orientación, motivación y ayuda por parte de su tutor durante la estadía. De los alumnos que recibieron apoyo por parte del tutor durante la estadía el 80\% se tituló.

Con base en las experiencias anteriores se pretende aplicar nuevamente la encuesta a la generación 2011-2013 para dar continuidad a la línea de investigación del cuerpo académico. Lo que se pretende con esta investigación es demostrar la importancia que tiene la acción tutorial del maestro en la culminación exitosa de la estadía del alumno y en consecuencia en la titulación, por lo que nuestra pregunta de investigación es la siguiente: ¿Influye en el índice de titulación la acción tutorial durante el período de estadía?

La presente investigación se considera viable por las siguientes razones: el equipo investigador labora actualmente en la UTCH, facilitando con esto el acceso a la información; se tiene registro de los egresados durante los 13 años que tiene de fundada la UTCH, además se dispone de datos en relación a las trayectorias académicas de los alumnos.

Este proyecto es de gran interés para la universidad, ya que con los datos que arroje esta investigación se detecta la importancia de la acción tutorial como factor que influye en el índice de titulación y se estará en posibilidad de tomar acciones correctivas para el logro del indicador.

\section{Antecedentes de Titulación en IES}

Varios países del mundo se han dado a la tarea de evaluar a las Instituciones de Educación Superior (IEs) con base en sus índices de titulación y eficiencia terminal. Algunos de ellos los miden a partir de la cohorte, es decir, los alumnos que ingresaron y lograron terminar sus estudios, otras los miden en función de los egresados y los que lograron obtener su título, encontrando en la mayoría de los casos que los porcentajes de estas mediciones no son los esperados.

La inquietud sobre este problema se manifestó desde principios de la década de los 
setenta y ha sido objeto de preocupación en diversos foros, entre los que destacan las Reuniones Nacionales de ANUIES en Villahermosa y Tepic, donde se planteó que una de las posibles causas de los bajos índices de titulación, podría deberse a la rigidez de los mecanismos de acreditación, tanto académicos como administrativos. De esas reuniones surgieron varias propuestas dirigidas a flexibilizar tales mecanismos para que existiera un mayor índice de titulación en las IEs.

En la investigación Un Modelo para medir la eficiencia terminal en las Universidades, Contreras Bolancé y Cuevas (2006) comentan que los problemas de eficiencia terminal suponen para el sistema educativo importantes pérdidas de recursos. El abandono de los estudios provoca un esfuerzo que finalmente no se traduce en la obtención de un título universitario. También, el retraso en la obtención del título es causa de ineficiencia en la asignación de recursos.

Por este motivo varias Universidades han llevado a cabo estudios que les ayuden a encontrar los factores que están influyendo para que estos índices no sean los esperados.

En la investigación que realizaron Melgar, Noreña, Plazola y Talavera(2006) mencionan que en promedio sólo el $50 \%$ de los alumnos de licenciatura y alrededor del $40 \%$ de los que cursan posgrados logran concluir estudios y titularse, lo que representa tanto un gasto de los recursos destinados a la educación como la frustración de legítimas aspiraciones personales. Los tiempos para lograr la titulación o gradua- ción son significativamente mayores y en la mayoría de las instituciones la diversidad de las opciones para la titulación es escasa y los procedimientos burocrático-administrativos constituyen un obstáculo.

En cuanto al problema de bajo índice de titulación se observa que no solo está relacionado con las opciones que tienen los estudiantes para terminar con sus estudios, sino también con algunos trámites y procedimientos ya sea administrativos, académicos o normativos (UABC, 2008).

Sin embargo, el problema no es solo de orden burocrático-administrativo sino que es necesario revisar y cuestionar aspectos académicos referentes a la formación que reciben los estudiantes durante su carrera profesional, es decir, habría que analizar si esa formación es lo suficientemente sólida para que el egresado pueda cubrir los requisitos académicos que la obtención del título exige, y de ser necesario, buscar nuevas opciones para demostrar los conocimientos, capacidades y habilidades de los estudiantes. Por otro lado, habría que tomar en cuenta también los factores exógenos, como por ejemplo la relación entre el fenómeno de la titulación y el mercado ocupacional dentro del contexto social actual (Pérez-Rocha, 1972).

Para poder entender la titulación y sus procesos habrá que enmarcarla como una manifestación del rendimiento dentro de un sistema escolar, en el nivel superior se han efectuado pocos estudios referentes al fenómeno de la titulación a lo largo de la vida de las diferentes universidades nacionales. Podemos ubicar, dentro de este contexto, a la Universidad Nacional Autónoma 
REVISTA DE INVESTIGACIÓN EDUCATIVA DE LA REDIECH N. 7 ISSN: 2007-4336

de México (UNAM), donde encontramos un primer antecedente de este tipo de trabajos. En uno de ellos, realizado por Garza (1986), se dió seguimiento a veintiún generaciones de estudiantes (1955-1975), desde su ingreso a la universidad hasta la obtención del grado. En el estudio se incluyen los porcentajes de titulación de doce facultades, a saber, Arquitectura, Ciencias, Ciencias Políticas y Sociales, Contaduría y Administración, Derecho, Economía, Filosofía y Letras, Ingeniería, Odontología, Psicología, Química y Veterinaria.

\section{Antecedentes de la tutoría}

En estudio realizado sobre el Impacto de la Tutoría en la Facultad de Estudios Superiores Zaragoza UNAM (2007), se menciona que en el año 2002 se implanta un programa de tutoría académica retomando las propuestas de la ANUIES y la Dirección General de Evaluación Educativa, con la finalidad de elevarlo al rango de institucional, el Programa de Tutoría se insertó en los Planes de Desarrollo 2000-2006 y en el 2006-2010 de la Facultad, como parte de los programas que pretenden mejorar el desempeño académico de los alumnos bajo la figura de un tutor, por medio de la atención personalizada y la formación integral, así como la retroalimentación al proceso de enseñanza-aprendizaje, el desarrollo de habilidades y hábitos de estudio, apoyo y orientación educativa.

El programa institucional de Tutoría de la FES-Zaragoza atiende aspectos cognitivos, afectivos y sociales de los alumnos de las siete licenciaturas de la Facultad, en el cual se considera a la tutoría: como un conjunto de actividades psicopedagógicas que tienen como propósito acompañar y apoyar a los alumnos durante su proceso de formación integral, orientándolos a partir del conocimiento de sus necesidades académicas, así como de sus inquietudes y aspiraciones profesionales. Promueve la disciplina en el trabajo y estimula la motivación del estudiante hacia su proceso formativo; fortalece sus habilidades para aprender a aprender y convertirse en aprendiz autónomo. Es una opción complementaria que no substituye las tareas del docente frente a grupo (Gómez, González y Palestino, 2010: 35).

\section{Antecedentes de la Tutoría en} el Subsistema de Universidades Tecnológicas

En las Políticas para la Operación, Desarrollo y Consolidación del Subsistema de Universidades Tecnológicas en el artículo $\mathrm{V}$ punto 67 , referente al personal académico se indica:

Los profesores de tiempo completo deberán distribuir sus 40 horas semanales de la siguiente manera: 15 horas en clase frente a grupo y 10 en proporcionar atención a los alumnos del grupo bajo su responsabilidad, conducir y supervisar sus estadías en la empresa y 15 a desarrollar materiales didácticos e investigación sobre los mismos (CGUT, 2002:17).

En el artículo X, Egreso y Titulación en el punto 115 indica que los requisitos que debe cumplir el egresado de cada Universidad Tecnológica para titularse son, además de la estadía, la presentación del informe de la misma (CGUT, 2002). 
En el punto 116 nos dice que las Universidades Tecnológicas bajo la responsabilidad del profesor de tiempo completo a cargo de cada grupo, deberá dar seguimiento y apoyo a los estudiantes que realizan su estadía para que avancen desde el primer momento, en la planeación y elaboración del informe sobre la misma (CGUT, 2002).

Al término de la estadía, el alumno tendrá un plazo de 15 días naturales para presentar ante el Director de Carrera el reporte debidamente requisitado, en original y copias simples (UTCH, 2005).

Con la constancia de que el reporte presentado fue satisfactorio, se consideran cumplidos los requisitos académicos para la obtención del título.

\section{Diseño metodológico}

El diseño metodológico de la investigación es descriptivo porque pretende medir y recoger información de manera independiente o conjunta sobre los conceptos o las variables a las que se refieren, así como los estudios exploratorios sirven fundamentalmente para descubrir y prefigurar, los estudios descriptivos son útiles para mostrar con precisión las dimensiones de la situación (Hernández Sampieri, 2010).

Es una investigación no experimental cuantitativa, ya que se puede definir como la investigación que se realiza sin manipular deliberadamente variables. Es decir, se trata de estudios donde no se manipulan en forma intencional las variables independientes para ver su efecto sobre las otras. Lo que hacemos en la investigación no experimental es observar fenómenos tal como se dan en su contexto natural para poste- riormente analizarlos. No se tiene control directo sobre dichas variables ni se puede influir sobre ellas, porque los fenómenos ya sucedieron, al igual que sus efectos. La investigación no experimental es un recurso de varios estudios cuantitativos, como son las encuestas de opinión, los estudios ex post facto retrospectivos y prospectivos. En esta investigación las inferencias sobre las relaciones entre variables se realizan sin intervención o influencia directa y dichas relaciones se observan tal como se ha dado en su contexto natural (Hernández, Fernández y Baptista, 2010).

Para efectos de esta investigación se diseñó una encuesta con la finalidad de conocer si la acción tutorial durante el período de estadía influye en el índice de titulación; para la realización de este estudio dicha encuesta se aplica al término del sexto cuatrimestre para recabar los datos necesarios.

La encuesta se compone de 10 preguntas: ¿Tu tutor te dio a conocer el programa de estadía? ¿Recibiste capacitación sobre el reporte de estadía? ¿Te hubiera gustado seleccionar a tu tutor de estadía? ¿Tu tutor te sugiere, orienta, motiva y ayuda durante el período de estadía? ¿Tu tutor te exige cumplir con el programa de estadía? ¿Hubo empatía con tu tutor de estadía? ¿El proyecto que se te asignó fue de tu agrado?, entre otras.

Se realizó una prueba piloto del cuestionario, el cual se aplicó a 10 maestros y 10 alumnos de la UTCH para revisar el nivel de redacción y comprensión de las preguntas incluidas en el instrumento, el resultado fue la identificación de los reactivos que eran ambiguos, los cuales se redactaron de 
nuevo para mejorar la comprensión de los mismos.

La aplicación del cuestionario se realizó en las instalaciones de la UTCH a una muestra de 53 alumnos de la generación 20112013 de la carrera de Mantenimiento Área Industrial.

Para fines de la captura de la información primero se enumeraron los cuestionarios alfabéticamente con la finalidad de ordenar e identificar cada sujeto entrevistado para validar la información.

El siguiente paso fue capturar los datos en el software estadístico para ciencias sociales (sPSs) y posteriormente compararlos con los resultados obtenidos de la generación 2007-2009 para detectar el impacto del acompañamiento del tutor durante el proceso de estadía y concientizar al mismo sobre la importancia que tiene su función en relación al índice de titulación.

Algunos de los resultados obtenidos en estudio previo de la generación 2007-2009 fueron:

En la pregunta que se les formuló a los alumnos si les hubiera gustado seleccionar a su tutor de estadía el $43 \%$ contestó que sí mientras el $56.4 \%$ que no.

El 92.3\% de los alumnos encuestados respondió que el tutor sí sugiere, orienta, motiva y ayuda durante la estadía y solo el 7.7\% indicó que no recibió sugerencias, orientación, motivación y ayuda por parte de su tutor durante la estadía.

El 96.2\% de alumnos encuestados contestó que el tutor sí le exige dar cumplimiento al programa de estadía y sólo $3.8 \%$ externó que no. Así mismo, el 87\% considera que sí hubo empatía con su tutor de estadía y sólo el $13 \%$ no se sintió bien con el tutor asignado.

De los alumnos encuestados el 91\% respondió que su tutor sí visitó la empresa donde realizó su estadía y sólo el 9\% contestó que su tutor no visitó la empresa.

Los datos obtenidos de la generación 2007-2009 de TSU se pretenden comparar con la actual generación 2011-2013 que cursa la estadía en el sector productivo.

\section{Resultados}

Observando la tabla 1 en donde se capturan los resultados que arrojaron las encuestas, se concluye que en general el acompañamiento que brinda el tutor en el proceso de estadía ha mejorado, solo hubo baja en la pregunta 8 ¿Tú tutor visitó la empresa en donde realizaste la estadía? bajó de un porcentaje del $91 \%$ al $81 \%$; en la pregunta 10 ¿Trabajas además de tus horas de estadía? Aumentó de un 19\% de alumnos que trabajaban a un 53\%.

Consideramos que esta es la causa por la cual de los 139 alumnos que egresaron solo 78 se titularon, lo cual equivale a un $56 \%$.

Respondiendo a nuestra pregunta de investigación ¿Influye en el índice de titulación la acción tutorial durante el período de estadía? se confirma que es importante dicho acompañamiento ya que en la generación 2007-2009 se tituló el 80\% de los alumnos egresados. Si lo analizamos por cohorte como lo mide la CGUTYP se tituló el 52\% estando por arriba de la media nacional, la cual es de 45\%. En la generación 
Tabla No. 1 Comparativa de resultados del estudio realizado sobre Análisis de la acción tutorial durante el proceso de estadía como factor que influye en el índice de titulación en la carrera de Mantenimiento Industrial de la UTCH entre la generación 2007-2009 y la generación 2011-2013.

\begin{tabular}{|c|c|c|c|c|c|}
\hline \multirow[t]{2}{*}{ No. } & \multirow{2}{*}{ PREGUNTA } & \multicolumn{2}{|c|}{$\begin{array}{c}\text { GENERACIÓN 2007- } \\
2009 \\
\% \\
\end{array}$} & \multicolumn{2}{|c|}{$\begin{array}{c}\text { GENERACIÓN } \\
2011-2013 \\
\% \\
\end{array}$} \\
\hline & & si & no & si & no \\
\hline 1 & ¿Tu tutor te dio a conocer el programa de estadía? & 95 & 5 & 100 & \\
\hline 2 & ¿Recibiste capacitación sobre el programa de estadía? & 92 & 8 & 100 & \\
\hline 3 & ¿Consideras complicados los trámites administrativos? & 40 & 60 & 41 & 59 \\
\hline 4 & ¿Te hubiera gustado seleccionar a tu tutor de estadía? & 43 & 57 & 43 & 57 \\
\hline 5 & ¿Tu tutor te sugiere, orienta, motiva y ayuda en la estadía? & 92 & 8 & 96 & 4 \\
\hline 6 & ¿Tu tutor te exige cumplir con el programa de estadía? & 96 & 4 & 98 & 2 \\
\hline 7 & ¿Hubo empatía con tu tutor de estadía? & 87 & 13 & 91 & 9 \\
\hline 8 & ¿Tu tutor visitó la empresa en donde realizaste la estadía? & 91 & 9 & 81 & 19 \\
\hline 9 & ¿El proyecto que se te asignó fue de tu agrado? & 95 & 5 & 98 & 2 \\
\hline 10 & ¿Trabajas además de tus horas de estadía? & 19 & 81 & 53 & 47 \\
\hline
\end{tabular}

2011-2013 aún no se obtienen los resultados finales por encontrarse en el proceso de titulación.

En 2014 se implementan las siguientes estrategias con el objetivo de titular al $100 \%$ de los egresados:

Se nombra un coordinador general de estadías en la carrera que será encargado de dar seguimiento a todo el proceso, iniciando desde febrero hasta noviembre y culmina con la ceremonia de graduación.

Se imparte curso del proceso de estadía a los alumnos del $5^{\circ}$ cuatrimestre próximos a egresar por parte del departamento de Vinculación y Secretaría Académica.

Adicionalmente la carrera de Mantenimiento Industrial ofrece seminario de titulación a todos los jóvenes próximos a egresar con el objetivo de reafirmar el proceso de estadía, se hace hincapié en los motivos por los cuales algunos de los compañeros no culminan su estadía quedando fuera del proceso de titulación.

Los alumnos de estadía se distribuyen a los maestros tutores de acuerdo al proyecto a realizar durante su estadía, y el perfil y experiencia del maestro.

Se distribuye vía electrónica a los alumnos y tutores los documentos oficiales de la estadía tales como: formato APA 6.0 para elaboración de reporte, calendario con fechas establecidas para revisión de documentos, formato oficial de autorización de proyecto, etc.

Los tutores de grupo brindan apoyo para localizar empresas en donde el alumno pueda realizar sus estadías.

Seguimiento personalizado de los tutores hacia los alumnos que cursan su estadía.

A los alumnos repetidores de estadía se les entrega minuta donde se explica que solo hay dos oportunidades de cursar la 
misma, quedando sobre aviso que si fallan quedan fuera de la UT y en consecuencia del proceso de titulación.

Estas acciones implementadas con la generación 2012-2014, podrán ser cuantificadas hasta el mes de septiembre en que finaliza el tiempo estipulado para titulación y estar en posibilidad de evaluar dichas estrategias.

\section{Referencias}

ANUIES. (2002). Programas institucionales de tutoría. Una propuesta de la ANUIES para su organización y funcionamiento en las instituciones de educación superior. Asociación Nacional de Universidades e Instituciones de Educación Superior (ANUIES). México. Consultado el 25/09/2010. Recuperado en: http:// www.anuies.mx/la_anuies/que_es/laanuies.php

CGUT. (2002). Políticas para la operación, desarrollo y consolidación del subsistema, en: http://cgut.utleon. edu.mx/images/stories/CGUT/cgut/politicaslineamientos.pdf

CGUT (2008-2009). Resultados del "Modelo de Evaluación de la Calidad del Subsistema de Universidades Tecnológicas". Consultado el 21/01/2011. Recuperado en: http://www.ses.sep.gob.mx/wb/ses/el_subsistema_de_universidades_tecnologicas_en

Contreras, A., Bolancé, C., Cuevas, E. (2006). Eficiencia terminal en las universidades, Expresión Económica, Revista de Análisis. julio-diciembre, 2006. Pp. 41-53. Universidad de Guadalajara. Centro de Investigacio- nes Sociales y Económicas. Ediciones de la noche.

Garza (1986). La titulación en la UNAM. Datos globales de titulación en: Books.google.com.mx/books?id=6 nASzDFfFcOC\&pg=PR5\&dq=L

Gómez, Y., González, M., Palestino, F. (2010). Programa Institucional de Tutorías Facultad de Estudios Superiores Zaragoza. http://www.tutoria.unam.mx/ EUT2010/memoriaEUT/htmls/memoria/HTMLS/ Eje5/GomezGutierrezYolanda.pdf

Hernández, R., Fernández, C., y Baptista, M. (2010). Metodología de la Investigación. 5a Edición. Mc Graw Hill. Chile.

Melgar, A., Noreña, S., Plazola, S. y Talavera, R. (2006). Factores que afectan la reprobación en estudiantes de la Facultad de Contaduría y Administración, UABC. Unidad Tijuana. Congreso Puebla, Puebla. México. Recuperado en: http://www.congresoretosyexpectativas.udg.mx/Congreso\%206/Eje\%202/Ponencia_82.pdf

Pérez Rocha, M. (1972). Algunos aspectos de la reestructuración académica de la enseñanza superior: cursos semestrales, salidas laterales y sistemas de titulación. Revista de la Educación Superior, Vol. 1. No. 4, Octubre-Diciembre.

UABC (2008). Indicadores de eficiencia terminal y titulación, Dirección de Planeación, UABC. Recuperado en: http://www.uabcs.mx/ddie/index.php?option= com_content \&view=article\&id=58\&itemid $=81$

UNAM (2010). Impacto de la tutoría en la FES Zaragoza, UNAM Evaluación 2007-2010, Recuperado en: www.tutoria.unam.mx/EUT2010/.../GomezGutierrezYolanda.pdf

UTCH (2005). Reglamento de Titulación. Recuperado en: www.utch.edu.mx 